\title{
Privacy or Convenience? The Current State and Future of Commercial Genetics
}

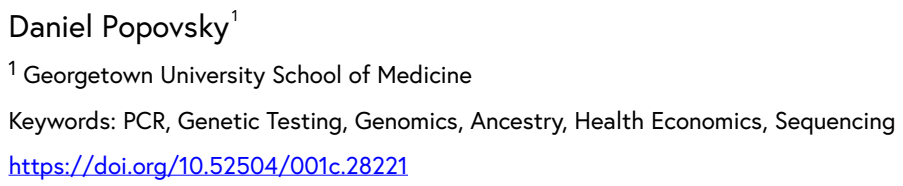

Georgetown Medical Review

Vol. 5, Issue 1, 2021

\section{Background}

Starting in 1990, the Human Genome Project set out with the ambitious goal to map the entire human genome. More specifically, the project's primary objective was to determine the nucleotide base pair composition of the geneassociated DNA in Homo sapiens - the segments involved in encoding functional proteins and regulatory segments. This information would help scientists better comprehend the pathophysiology of numerous diseases, and potentially spark a new era of genetically targeted treatment. ${ }^{1}$ The cost of the project amounted to nearly $\$ 3$ billion. Thirty years later, in 2020, technological advances in large-scale gene sequencing allow a patient to have his or her whole genome sequenced for less than $\$ 100 .^{2}$ Various forms of genetic testing-including whole exome sequencing and microarray studies to ascertain copy number variants-are therefore now widely accessible. They have become, in effect, a bedrock of clinical testing among the broader menu of laboratory analyses available in a well-equipped hospital or clinic, capable of being ordered right alongside a blood smear or urinalysis.

Not only have genetic testing tools become more accessible in the clinical setting, but they are also available to consumers even outside of clinical applications entirely. Companies like $23 \&$ me and Ancestry.com have provided services for customers to access their own genome and learn more about their ancestry, phenotypes, and risks for many prevalent diseases. While this widespread access has no doubt expanded our understanding of our species and ability to treat disease, there are also salient ethical considerations that must be discussed when one's genome is being sequenced and analyzed by a third party. This paper will focus on the benefits and risks of the genomic data mining enterprise, as well as how it will impact consumers and society as a whole for years to come.

Direct-to-consumer genetic testing has had a marked impact on the average individual's ability to discover and learn about their overall health, genetic background, and potential medical predispositions. At the same time, there has been comparatively sparse awareness surrounding the implications that a genetic disorder can have, and how complex gene interactions can modulate the development of multifactorial chronic conditions. A 2011 study on genetic 
disease awareness sampled 1,747 households on whether or not they believed genetics influenced chronic disease. Findings of this survey were mixed, with many unaware or doubtful of the impact of relevant genes on their overall wellbeing; however, those who recognized genetics as a factor for chronic disease progression were more likely to also acknowledge the importance of lifestyle in modulating the genes' effects, and how better habits can tangibly impact the progression of chronic disease, thereby embracing lifestyle changes and better overall health outcomes. ${ }^{3}$ At-home genetic testing delivers, in essence, an ability to become aware of one's own genetic predispositions which, if interpreted correctly, can encourage individuals and families to be more proactive and salutary in their lifestyle choices.

Direct-to-consumer genetic testing also offers distinct advantages in the realm of cost and affordability. A barrier to many clinical screening tests is the difficulty in receiving approval from insurance companies, which are often indisposed to covering their full costs. The economical price of mass-market tests $-\$ 91$ for Ancestry. Com and $\$ 99$ for $23 \&$ me-without need for insurance approval, allows for consumers to be tested and procure results without having to concern themselves with third-party coverage. Overall, in fact, genetic testing through a healthcare provider can be more expensive than buying a testing kit (or sending out samples) directly from a providing company. ${ }^{4} \mathrm{On}$ a societal level, the aggregate genetic information gathered from these commercial outfits can help further genetic research, and even expedite identification of pathological genes and suitable genetic targets for pharmaceutical intervention. Consumers can also ascertain their ancestral background and in some cases, discover relatives that they may not have known about previously. All of this information can be gathered and analyzed quickly, from the comfort of one's own home.

\section{Implications}

As with many disruptive technological developments, nevertheless, their impact can be a double-edged sword. While there are undoubtedly numerous benefits of direct-to-consumer genetic testing, there is very little rigorous oversight or regulation of the companies that sell these tests and store an individual's genomic data. A person's genome is, in essence, a very detailed and powerful resource for a variety of reasons. First, one's genetic information furnishes a background of many features that paint the picture of a unique individual. Ancestry, for instance, may concomitantly reveal details about one's culture, location, and physical phenotype- traits that are physically expressed and detectable such as hair, skin, and eye color. Second, and more significantly, one's genome reveals health conditions that a person may currently have, or potentially will have in the future.

Such information altogether can be used as an informatics-driven platform, in effect to develop marketing tools utilized by social media, pharmaceutical companies, or even insurance companies. If a person for example has the 
phenotype for fair skin, a company can use this information to market specific skin care products to that individual. A potentially more problematic and even dangerous aspect of this knowledge, however, is its potential to influence and, especially, to restrict or hamper one's healthcare options. This is particularly true in the United States and other countries that lack universal health care, where an individual with a potentially pathogenic genetic variant, predisposing them to a serious illness (like Huntington's Disease or Alzheimer's), can have such information be used against them. They can, in effect, fall victim to having their genetic record adversely impact insurance premiums, or even job prospects.

Genetic testing companies like 23\&me and Ancestry.Com are aware of these concerns and have attempted to address them head-on. ${ }^{5}$ Both enterprises have explicit privacy policies, expressly stating that genomic data will not be shared with public databases, or with insurance companies and employers without the consumer's consent. ${ }^{5}$ It is most crucial to note here that, while a consumer may opt to not have their data shared with third parties, nevertheless the testing company, not to mention its own corporate investors, contingent upon the investment terms, may have comprehensive access to this data. Corporate access to this type of data, by way of investment into the original corporation, is a concept known as ghost access. ${ }^{6}$ This in practice creates a sort of "back door", potentially allowing third parties to gain access to protected data without technically breaching privacy policies. As recently as last year, GlaxoSmithKline, one of the world's biggest pharmaceutical companies, invested $\$ 300$ million into $23 \& \mathrm{me}^{7}$ This investment lent GSK access to $23 \&$ me's vast collection of genomic data collected from the firm's consumers, facilitating the development of a new drug for psoriatic arthritis. ${ }^{8}$ To compound this thorny issue, $23 \& \mathrm{me}$ also mentions, in its terms of service, that consumers who allow their genetic data to be used will not see any financial gain from drugs developed from their genomes. ${ }^{5}$ Instances like this show that, despite even the most explicit privacy policies, companies can nonetheless introduce what amounts to fine print, in a way that allows consumers' genetic information to be used in ways that are quite lucrative for the firms gathering such data. Practical and ethical questions therefore arise, inevitably, regarding the potential for this information to be used for applications outside of drug research per se.

\section{Policitcal Action}

The United States government has taken some action in order to address the potential discrimination and privacy issues inherent in the direct-to-consumer genetic testing industry. The Genetic Information Non-Discrimination Act (GINA), for example, was passed in 2008 so as to preclude the use of genetic information in health insurance and employment decisions (U.S Equal Employment Oppurtunity Commision). Nevertheless, this law does not cover life, disability, and long-term health insurance, and thus its scope is limited (U.S Equal Employment Oppurtunity Commision). A significant additional 
drawback to this law is that it does not enhance measures that promote personal control over one's genetic testing results. This allows companies such as $23 \&$ me to use one's genetic information for their own benefit, in various ways as laid out above. Moreover, with greater accessibility of the results of genetic testing, efforts have already been made to dilute and weaken the protections afforded by GINA. In 2017 a bill, HR 1313, was introduced which would have allowed employers to demand workers' genetic testing results. While this bill was ultimately rejected, its very introduction into a Congressional committee is a clear sign that institutions, both public and private, have come to recognize the tangible value of genetic testing, unfortunately often in ways that can be used against the interests of the person who requests the testing. If protections erode for people's genetic information, then concerning scenarios like those mentioned before become more plausible. This could have devastating and detrimental impacts on the lives of Americans, including the imposition of financially ruinous health insurance premiums, or even losing a job due to a concerning genetic predisposition.

\section{Ethical Considerations}

With the sweeping changes genetic testing is bringing to healthcare and sociocultural systems, it is therefore essential to acknowledge the ethical considerations and imperatives that accompany such new technology. The dilemmas surrounding these considerations, and possible solutions for them, can be framed in part through the lens of patient and consumer agency, and the broader doctor-patient relationship. Dr. Edmund Pellegrino, in his writings on this relationship, places an emphasis on patient autonomy in particular as a bulwark against misuse of an individual's health data. ${ }^{9}$ More specifically, he refers to an autonomy-based system that includes two models of the doctorpatient relationship: the consumer model and the negotiated contracts model. In the former, medicine as a whole is viewed as a commodity, with the physician providing tangible value via knowledge and specialized, targeted treatment. In the latter model, a patient and a physician enter a sort of negotiated contract, in which the terms of care are pre-determined. Underlying both models is a conviction that patients have full agency in accepting (or rejecting) treatment recommendations, and in permitting or refusing access to any data collected in the course of treatment. ${ }^{10}$

These defining models, which frame the patient-physician relationship on a granular level with the presumption of two individuals in mind, must be significantly adjusted as patients' relationships shift away from interactions with a single individual or small group, to one with private companies that provide novel medical services. In the case of genetic testing, do the traditional principles of autonomy, as outlined by Dr. Pellegrino, still apply? Should a person's genetic information, maintained within the databases and physical servers of a company with distant headquarters, be thought of as an extension of themselves, and therefore still be bound by the principles of autonomy? We can easily apply the two models of an autonomy-based system, as above, in 
the context of a person-person relationship to fully justify such constraints. However, it is also possible to supply and accept frameworks which suggest a different interpretation in regard to the companies that handle a person's genetic info.

The consumer relationship, as entered into with mainstream genetic testing companies, can align with both the consumer model and negotiated contract models as outlined by Dr. Pellegrino. They provide consumers with a defined medical service-the specialized analysis of one's genome-in exchange for a predetermined price. Additionally, when agreeing to work with a genetic testing company, like $23 \& \mathrm{me}$, one is entering into a pre-determined contractual agreement. However, unlike the contract made with a physician, where one agrees to terms of care that is temporally and spatially confined to the clinical center where treatment occurs, direct-to-consumer genetic testing implies an agreement to have one's genetic information indefinitely stored within the databases of a third party. Moreover, while HIPAA and other federal and state statutes (and court decisions) tightly control the use of stored patient information in a hospital or other clinical context, genetic testing firms may not be bound by such constraints, at least not in the same ways. ${ }^{11}$ As part of their contracts, consumers approve the terms of how said company handles collected information, which may include directly profiting from it without compensating (or even informing) the individual who contributed the data. We must ask ourselves to what extent a genome constitutes, or is legally and practically tantamount to, a patient- and whether or not an individual should have greater control over how their genetic data is being processed.

Ultimately, these considerations shine an unflattering and somewhat concerning light on the power that genetic testing companies have accumulated by virtue of the personal data they are collecting on potentially millions of consumers, and the relatively lax oversight and regulation of how that data can be used.

In effect, the standard expectations of patient autonomy and agency in regard to clinical information, as laid out by Pellegrino and many others, are more loosely interpreted and fungible in the context of data collection by firms that specialize in the gathering and interpretation of individuals' genetic sequence data. This is in part because the notion of the contract in the doctor-patient relationship, which exists principally in an implied form, takes on a more explicit character when genetic testing firms extract saliva, blood, or tissue samples-and the precious haul of genetic data housed within them. The disruptive nature of the genetic testing industry has resulted in contractual arrangements that, in their literal reading, effectively sign away many of the patient's rights to (and control over) data, that are considered elemental to the traditional doctor-patient or nurse-patient relationship. In effect, clients of these companies are often waiving these rights without full awareness of the 
implications of such actions, a problem exacerbated in part by the apparently benign ramifications of this decision up-front, but also by the uncertainty of a distant future where the application of such genetic data remains unclear.

\section{Conclusion}

At root, the danger of poorly-regulated genetic information collection lies on a slippery slope, and far away from initial intentions or interests. Even though assembled genetic data may not be put to nefarious use shortly after the time of collection, it remains a commodity in the indefinite hold of not only the firm which obtains it, but also any major investors or partners who may, through flexible interpretations of laws or loose regulations, eventually gain hold of it. This includes institutions in both the public and private sector. It is therefore critical to provide full disclosure to clients about these possibilities and, more importantly, to proactively establish standards, conventions, and protections up-front that safeguard genetic data indefinitely into the future, while also establishing ground rules for allowing patients to maintain agency over critical decisions involving the use of said data. Ultimately, the guiding principle for the utility of such data should likely be directed by the same principle that steers virtually every other decision in medicine-first, do no harm. Genetic data is a valuable resource, but ultimately one that should be used for the collective good of both the individual and the community of which that individual is a part. It should never be exploited for the gain of outside parties at the expense of the individual who provides it. Such basic tenets are at the heart of not only medical ethics but also the broader societal ethics, associated with the foundational ethical teachings of Immanuel Kant, John Locke, and JeanJacques Rousseau-which underpin the very idea of the social contract, and a rights-based society in general. They should not be conveniently set aside simply due to the rise of a new and disruptive technology. 


\section{REFERENCES}

1. Lander ES et al. Initial Sequencing and Analysis of the Human Genome. Nature. 2001;409(6822):860-921.

2. How Much Does an AncestryDNA® Test Cost? Accessed January 18, 2021. https://www.ancestry.com/dna/lp/ancestry-dna-cost

3. Genetic Information Discrimination. Accessed January 18, 2021. https://www.eeoc.gov/geneticinformation-discrimination

4. What Are the Benefits and Risks of Direct-to-Consumer Genetic Testing? Accessed January 18, 2021. https://medlineplus.gov/genetics/understanding/dtcgenetictesting/dtcrisksbenefits/

5. 23andMe. DNA Genetic Testing \& Analysis - 23andMe. Accessed January 18, 2021.

https://www.23andme.com/about/privacy/

6. Hunter P. The Big Health Data Sale: As the Trade of Personal Health and Medical Data Expands, It Becomes Necessary to Improve Legal Frameworks for Protecting Patient Anonymity, Handling Consent and Ensuring the Quality of Data. EMBO Rep. 2016;17(8):1103-1105. doi:10.15252/embr.201642917

7. Ducharme J. A Major Drug Company Now Has Access to 23andMe's Genetic Data. Should You Be Concerned? Time. Published online July 2018. https://time.com/5349896/23andme-glaxosmith-kline/

8. Wetsman N. 23andMe Sold the Rights to a Drug It Developed from Its Genetic Database. The Verge. Published online January 10, 2020. https://www.theverge.com/2020/1/10/21060456/ 23andme-licensed-drug-developed-genetic-database-autoimmune-psoriasis-almirall

9. Pellegrino ED. The Commodification of Medical and Health Care: The Moral Consequences of a Paradigm Shift from a Professional to a Market Ethic. The Journal of Medicine and Philosophy. 1999;24(3):243-266. doi:10.1076/jmep.24.3.243.2523

10. Pellegrino ED. The Four Principles and the Doctor-Patient Relationship: The Need for a Better Understanding. Published online 1994. https://repository.library.georgetown.edu/handle/10822/ $\underline{712079}$

11. National Research Council, et al. Direct-to-Consumer Genetic Testing: Summary of a Workshop. National Academies Press; 2010. 